\title{
ON THE NACHBIN UNIFORM STRUCTURE
}

\author{
NELSON ONUCHIC
}

Let $\left(\mathcal{U}_{\alpha}\right)$ be the set of uniform structures compatible with the topology of a completely regular space $E$. The least fine $\mathfrak{u}_{\alpha}$, that makes all real-valued continuous functions uniformly continuous, is called the Nachbin uniform structure. It is denoted by $\mathfrak{u}_{N}(E)$. We present here the following questions:

If $E$ is a completely regular space, is the equality $\mathfrak{u}_{N}(E \times E)$ $=\mathfrak{u}_{N}(E) \times \mathfrak{u}_{N}(E)$ valid? For a compact space $E$, that equality is valid. This is obvious enough not to require a proof. For a noncompact space $E$, that equality is in general not valid. In this article we will give a partial answer to this question. We will show that in the most interesting cases (except that of the compact spaces), the answer is negative.

We have the finest uniform structure compatible with the topology of $E$. It is denoted by $\mathcal{U}_{0}(E)$, and the following is true:

If $\mathcal{u}(E)$ is a uniform structure on $E$ such that every continuous and finite écart on $E$ is uniformly continuous on $E \times E$ with the uniform structure $\mathfrak{u}(E) \times \mathfrak{u}(E)$, then $\mathfrak{u}(E)=\mathfrak{u}_{0}(E)$.

Proposition 1. Let $E$ be a completely regular space. If $\mathfrak{u}_{N}(E \times E)$ $=\mathfrak{u}_{N}(E) \times \mathfrak{u}_{N}(E)$, then $\mathfrak{u}_{N}(E)=\mathfrak{U}_{0}(E)$.

Proof. Every real-valued continuous function of $E \times E$ with $\mathfrak{u}_{N}(E \times E)$ is uniformly continuous. As $\mathfrak{u}_{N}(E \times E)=\mathfrak{u}_{N}(E) \times \mathfrak{u}_{N}(E)$ then every continuous and finite écart on $E$, with $\mathfrak{u}_{N}(E) \times \mathfrak{u}_{N}(E)$, is uniformly continuous. Then, $\mathfrak{u}_{N}(E)=\mathfrak{u}_{0}(E)$.

PROPOSITION 2. Let $E$ be a completely regular space. If $\mathfrak{u}_{N}(E)$ $=\mathcal{U}_{0}(E)$, then every open partition of $E$ is countable.

Proof. Tukey has shown that each uniform structure on $E$ is determined by the family of uniform coverings of $E$ in this structure [9]. Shirota proved Tukey's conjecture that the family of all countable normal coverings of $E$ is always the family of uniform coverings for a uniform structure $\mathcal{u}_{e}(E)$ [8]. Evidently $\mathcal{u}_{e}(E)$ is finer than $\mathfrak{u}_{N}(E)$. On the other hand, the uniform coverings for $\mathfrak{u}_{0}(E)$ are precisely the normal coverings [9], and every open partition is trivially normal. Thus if $\mathfrak{U}_{0}(E)=\mathfrak{U}_{N}(E)$, then every open partition must be countable.

CoRollary. Let $E$ be a completely regular space such that $\mathfrak{u}_{N}(E)$ $\times \mathfrak{u}_{N}(E)=\mathfrak{u}_{N}(E \times E)$. Then, every open partition is countable.

Received by the editors February 11, 1959. 
With the purpose of arriving at the Theorem and the subsequent considerations which will give us very interesting information for our problem, we will make a preliminary preparation.

A completely regular space $E$ is called a $P$-space if every countable intersection of open sets of $E$ is open. A completely regular space $E$ is a $P$-space if, and only if, for all $f \in C(E, R), f^{-1}(0)$ is open [3, Theorem 5.3].

We define the following uniform structure compatible with the topology of a $P$-space $E$ :

For every real-valued continuous function we define $U_{f}=U_{r \in R} f^{-1}(r)$ $\times f^{-1}(r)$. The family $\left(U_{f}\right)_{f \in C(E, R)}$ is a base for a filter for a uniform structure compatible with the topology of $E$. This uniform structure is called the natural uniform structure. It is denoted by $\mathfrak{u}_{P}(E)$ [5].

Proposition 3. Let E be a P-space and $E \times E$ a Lindelöf space, i.e., each open covering of $E \times E$ has a countable subcovering. Then,

$$
\mathfrak{u}_{N}(E \times E)=\mathfrak{u}_{N}(E) \times \mathfrak{u}_{N}(E) .
$$

Note. There is a nontrivial $P$-space, i.e., different from a discrete space and such that the hypotheses of the Proposition 3 are satisfied. Let $E$ be a set of cardinal number equal to $\boldsymbol{\aleph}_{1}$ and $x \in E$. We define on $E$ the following topology: for $x$ the filter of neighborhoods is given by all sets $V$ such that $x \in V$ and $E-V$ is countable and all the points different from $x$ are open. The set $E$ with this topology satisfies the hypotheses of the proposition above.

Proof of the Proposition 3. As $E$ is a $P$-space and a Lindelöf space, then $\mathfrak{u}_{N}(E)=\mathfrak{u}_{P}(E)$ [6, Proposition 3]. As $E \times E$ is a Lindelöf space $\mathfrak{u}_{P}(E \times E)=\mathfrak{u}_{P}(E) \times \mathfrak{u}_{P}(E)$ [6, Proposition 4]. This implies $\mathfrak{u}_{N}(E \times E)=\mathfrak{u}_{N}(E) \times \mathfrak{u}_{N}(E)$.

A completely regular space is called a pseudo-compact space if all real-valued continuous functions are bounded.

THEOREM. Let $E$ be a completely regular space. In order that $\mathfrak{u}_{N}(E \times E)$ $=\mathfrak{U}_{N}(E) \times \mathfrak{U}_{N}(E)$, it is necessary that $E$ be a pseudo-compact space, or a $P$-space such that every open partition is countable.

Proof. Let $E$ be a completely regular space non- $P$-space and nonpseudo-compact space. There exists a real-valued continuous nonbounded function $f$ and $x_{0} \in E$ such that $f$ is nonconstant on every neighborhood of $x_{0}$. Let us show that the continuous function $g: g(x, y)$ $=f(x) \cdot f(y)$, defined on $E \times E$, is nonuniformly continuous with respect to the uniform structure $\mathfrak{U}_{N}(E) \times \mathfrak{U}_{N}(E)$. This implies that $\mathfrak{u}_{N}(E) \times \mathfrak{u}_{N}(E) \neq \mathfrak{u}_{N}(E \times E)$.

Let $V$ be any entourage of the filter of entourages of $\mathfrak{u}_{N}(E)$ and 
$x \in E$ such that $\left(x_{0}, x\right) \in V$ and $f(x) \neq f\left(x_{0}\right)$. Since $f$ is not bounded, $\left|f(x) \cdot f(y)-f\left(x_{0}\right) \cdot f(y)\right|=|f(y)| \cdot\left|f(x)-f\left(x_{0}\right)\right|$ can be greater than any given number, for a convenient choice of $y$. Therefore $g$ is not uniformly continuous. If $\mathfrak{u}_{N}(E \times E)=\mathfrak{u}_{N}(E) \times \mathfrak{u}_{N}(E)$ and $E$ a $P$-space, then, it results from the corollary that every open partition of $E$ is countable.

Note. The hypotheses of the above theorem are not sufficient. In fact, if $E$ is a pseudo-compact space the problem is equivalent to knowing whether $\beta(E \times E)=\beta(E) \times \beta(E)$, where $\beta(X)$ is the StoneCech compactification of a completely regular space $X$. We know that $\beta(E \times F)=\beta(E) \times \beta(F)$ and $E$ and $F$ infinite implies that $E \times F$ is a pseudo-compact space [4]. There are countably compact spaces $A, B$ (hence $A, B$ are pseudo-compact spaces), such that $A \times B$ is not pseudo-compact [7]. Then, $E=A \cup B$ is pseudo-compact and $E \times E$ is not pseudo-compact. This shows that $\beta(E \times E) \neq \beta(E)$. I. Glicksberg's paper Cech compactifications of products ${ }^{1}$ proved that $\beta(E \times F)$ $=\beta(E) \times \beta(F)$ if and only if $E \times F$ is a pseudo-compact space. BagleyConnell-McKnight gave a sufficient condition for a product space to be pseudo-compact [1, Theorem 6 and Corollary].

We can say that if $E$ is a $P$-space such that $E \times E$ is a Lindelöf space, then by the Proposition 3 , the condition of the above theorem is sufficient.

We wish to thank the referee for the suggestions and references given in the proof of the Proposition 2 and in the last note.

\section{BIBLIOGRAPHY}

1. R. W. Bagley, E. H. Connell and J. D. McKnight, On properties characterizing pseudo-compact spaces, Proc. Amer. Math. Soc. vol. 9 (1958) pp. 500-506.

2. N. Bourbaki, Topologie générale, Chapters I and II, 2d ed., Paris, Hermann, 1951.

3. L. Gillman and M. Henriksen, Concerning rings of continuous functions, Trans. Amer. Math. Soc. vol. 77 (1954) pp. 340-362.

4. M. Henriksen and J. R. Isbell, On the Stone-Čech compactification of a product of two spaces, Bull. Amer. Math. Soc. Abstract 63-2-332.

5. C. S. Honig, Sur quelques propriétés des P-espaces, An. Acad. Brasil. Ci. vol. 29 no. 3 (1957) pp. 319-322.

6. N. Onuchic, P-spaces and the Stone-Čech compactification, An. Acad. Brasil. Ci. vol. 30 no. 1 (1958) pp. 43-45.

7. J. Novak, On the cartesian product of two compact spaces, Fund. Math. vol. 40 (1953) pp. 106-112. $23-40$.

8. T. Shirota, A class of topological spaces, Osaka Math. J. vol. 4 no. 1 (1952) pp.

9. J. W. Tukey, Convergence and uniformity, Princeton, University Press, 1940.

Ciências e Letras de Rio Claro, Sao Paulo, Brasil

1 Published in Trans. Amer. Math. Soc. vol. 90 (1959) pp. 369-382. 